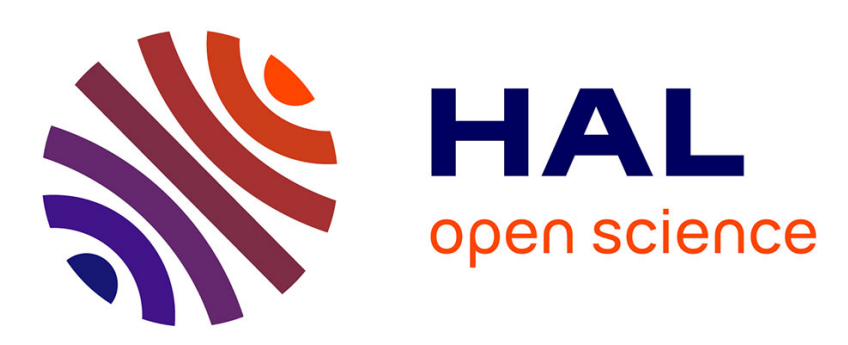

\title{
Improved Balanced 2n-PSK STTCs for Any Number of Transmit Antennas from a New and General Design Method
}

Pierre Viland, Gheorghe Zaharia, Jean-François Hélard

\section{To cite this version:}

Pierre Viland, Gheorghe Zaharia, Jean-François Hélard. Improved Balanced 2n-PSK STTCs for Any Number of Transmit Antennas from a New and General Design Method. Vehicular Technology Conference, 2009. VTC Spring 2009. IEEE 69th, Apr 2009, Barcelone, Spain. pp.1-5, 10.1109/VETECS.2009.5073590 . hal-00429915

\section{HAL Id: hal-00429915 https://hal.science/hal-00429915}

Submitted on 5 Nov 2009

HAL is a multi-disciplinary open access archive for the deposit and dissemination of scientific research documents, whether they are published or not. The documents may come from teaching and research institutions in France or abroad, or from public or private research centers.
L'archive ouverte pluridisciplinaire HAL, est destinée au dépôt et à la diffusion de documents scientifiques de niveau recherche, publiés ou non, émanant des établissements d'enseignement et de recherche français ou étrangers, des laboratoires publics ou privés. 


\title{
Improved Balanced $2^{n}$-PSK STTCs for Several Transmit Antennas from a New and General Design Method
}

\author{
Pierre Viland, Gheorghe Zaharia, Jean-François Hélard \\ Institute for Electronics and Telecommunications of Rennes (IETR) - UMR CNRS 6164 \\ INSA - 20 avenue des Buttes de Coësmes, 35043 Rennes, France \\ Email: pierre.viland@insa-rennes.fr
}

\begin{abstract}
Recently, it has been established that the best spacetime trellis codes (STTCs) belong to a specific class of codes. These codes are called "balanced STTCs" because they use the points of the MIMO constellation with the same probability. Therefore, the search of the best codes can be reduced to this class. This paper presents a new and general method to design $2^{n}$. PSK balanced STTCs for any number of transmit antennas. This method is simpler than the first method, which was described only for 4-PSK modulation and can be generalized for any configuration of the space-time trellis encoder. Simulation results of new 4-PSK and 8-PSK balanced codes prove the importance of this class.
\end{abstract}

Keywords - space-time trellis codes, balanced codes, MIMO systems, design method.

\section{INTRODUCTION}

The concept of space-time trellis codes (STTCs) was proposed in 1998 by Tarokh et al. [1] by combining timeconvolution coding with multiple transmit antennas to improve the data rate and the reliability of wireless communication. In [1], the rank and the determinant criteria were proposed to design STTC in the case of slow fading channel. It was shown in [2] that, for a great product of the number of transmit and receive antennas, the performance for a slow fading channel is determined by the trace criterion.

Based on the above criteria, many codes for 2, 3 and 4 transmit antennas have been proposed [3-7] after a systematic search to decrease the bit and frame error rate. In [8], it has been remarked that the codes achieving the best performance have the same property: the used points of multiple input multiple output (MIMO) constellation are generated with the same probability. Therefore, these codes are referred to as "Balanced space-time trellis codes: B-STTCs" [8-10].

The aim of this paper is to present a general method to design the class of balanced $2^{n}$-PSK STTCs which offers the best performance. Therefore, the systematic search for good codes can be reduced to this class. The rest of this paper is organised as follows. In section 2, the encoder is briefly described. The performance criteria of STTCs are presented in section 3. Section 4 presents the main properties of the balanced codes. In section 5, the general method to design balanced codes is described for any number of transmit antennas and for $2^{n}$-PSK modulations. Some examples to design balanced codes for specific configurations are given. Finally, some new balanced codes which outperform the best known codes are proposed for 4-PSK and 8-PSK modulations.

\section{SPACE Time TRELlis CODING}

We consider the general case of the $2^{n}$-PSK space-time trellis encoder. Fig. 1 shows the encoder for $n=2$.

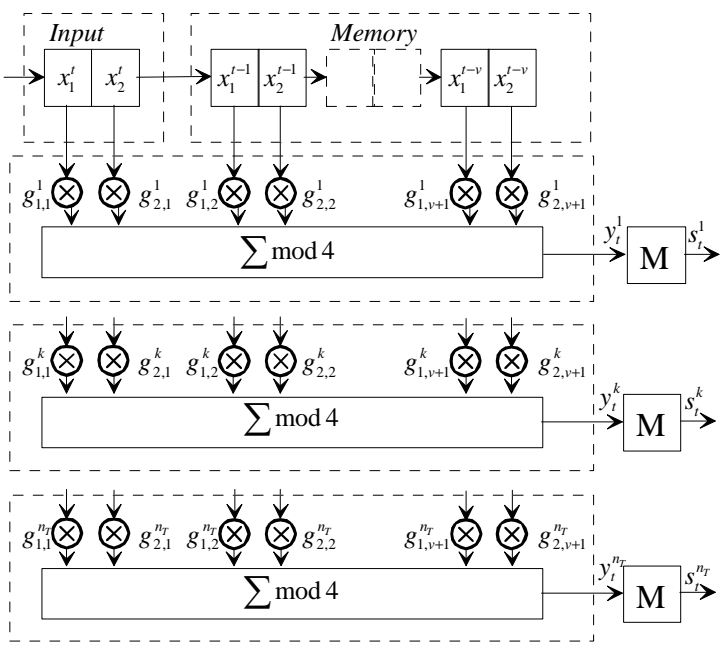

Fig. 1. 4-PSK space time trellis encoder with $n_{T}$ transmit antennas

This encoder has one input block of $n$ bits and $v$ memory blocks of $\mathrm{n}$ bits. At each time $t \in \mathbb{Z}$, the $n$ bits of a block are replaced by the $n$ bits of the previous block. Usually, the state of the encoder is defined by the binary values of $v$ memory blocks of $n$ bits. The $i^{t h}$ bit $x_{i}^{t-j+1}, i=1$. . n of the $j^{\text {th }}$ block, $j=1 \ldots(v+1), \quad$ is associated to $n_{T}$ multiplier coefficients $g_{i, j}^{k} \in \mathbb{Z}_{2^{n}}, k=1 \ldots n_{T}$, where $n_{T}$ is the number of transmit antennas. A space-time trellis encoder is thus usually defined by its generator matrix $\boldsymbol{G}$ of $n_{T} \times n(v+1)$ coefficients

$$
\boldsymbol{G}=\left[\begin{array}{ccccccc}
g_{1,1}^{1} & \ldots & g_{n, 1}^{1} & \ldots & g_{1, v+1}^{1} & \ldots & g_{n, v+1}^{1} \\
\vdots & & \ldots & & \ldots & & \vdots \\
g_{1,1}^{k} & \ldots & g_{n, 1}^{k} & \ldots & g_{1, v+1}^{k} & \ldots & g_{n, v+1}^{k} \\
\vdots & & \ldots & & \ldots & & \vdots \\
g_{1,1}^{n_{T}} & \ldots & g_{n, 1}^{n_{T}} & \ldots & g_{1, v+1}^{n_{T}} & \ldots & g_{n, v+1}^{n_{T}}
\end{array}\right]
$$


The encoder outputs for the $k^{\text {th }}$ antenna are computed as

$$
y_{t}^{k}=\sum_{i=1}^{n} \sum_{j=1}^{v+1} x_{i}^{t-j+1} g_{i, j}^{k} \quad \bmod 2^{n}
$$

Each encoder output $y_{t}^{k}$ is mapped onto a $2^{n}$-PSK signal given by: $s_{t}^{k}=\exp \left(j \frac{\pi}{2^{n-1}} y_{t}^{k}\right)$. Each output signal $s_{t}^{k}$ is send to the $k^{\text {th }}$ transmit antenna. The modulated streams of all antennas are then transmitted simultaneously.

\section{DESIGN CRITERIA}

Several design criteria have been proposed in $[1,2]$ to exploit the spatial diversity and to offer optimal coding gain. The case of slow Rayleigh fading propagation channels is mainly considered in this paper. The $n_{T}$ dimension transmitted symbols $\boldsymbol{s}_{\boldsymbol{t}}=\left[s_{t}^{1} s_{t}^{2} \ldots s_{t}^{n_{T}}\right]^{T}$, where $[\cdot]^{T}$ denotes the transpose operator, are assumed to be grouped in a frame of length $L_{f}$. Traditionally, criteria are derived from the minimization of the pairwise error probability (PEP), i.e. the probability of transmitting the $n_{T} \times L_{f}$ dimension coded frame $S_{t}=\left[s_{t} s_{t+1} \ldots s_{t+L_{f}-1}\right]$ and deciding erroneously in favour of another $n_{T} \times L_{f}$ dimension coded frame

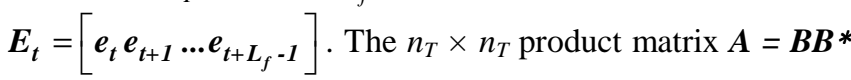
is introduced, where $\boldsymbol{B} *$ denotes the hermitian of the $n_{T} \times L_{f}$ difference matrix $\boldsymbol{B}=\boldsymbol{E}_{\boldsymbol{t}}-\boldsymbol{S}_{\boldsymbol{t}}$.

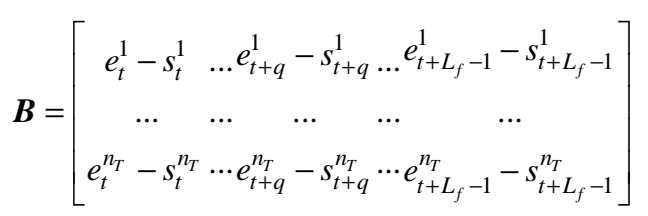

Let us defined $n_{R}$ the number of receive antennas. In order to achieve the minimum error probability, if $\min (\operatorname{rank}(\boldsymbol{A})) n_{R} \leq 3$ and for slow fading channels, we should maximize the minimum rank and the minimum determinant of the matrix $\boldsymbol{A}$ computed for all pairs of coded frames $\left(\boldsymbol{E}_{t}, \boldsymbol{S}_{t}\right)$ [1].

In [2], Chen proposed a new criterion which is valid for slow and fast Rayleigh fading channels if $\min (\operatorname{rank}(\boldsymbol{A})) n_{R}>3$. Under this assumption, the PEP is minimized if the sum of all the eigenvalues of the product matrix $\boldsymbol{A}$ is maximized. Because $\boldsymbol{A}$ is a square matrix, the sum of all the eigenvalues $\lambda_{k}$ is equal to the trace of the matrix $A$

$$
\operatorname{tr}(\boldsymbol{A})=\sum_{k=1}^{n_{T}} \lambda_{k}=\sum_{k=1}^{n_{T}}\left(\sum_{q=0}^{L_{f}-1}\left|e_{t+q}^{k}-s_{t+q}^{k}\right|^{2}\right)
$$

For each pair of coded frames, the matrix $\boldsymbol{A}$ and then $\operatorname{tr}(\boldsymbol{A})$ can be computed. The minimum trace is the minimum of all these values $\operatorname{tr}(\boldsymbol{A})$. When the product $\min (\operatorname{rank}(\boldsymbol{A})) n_{R}>3$, the minimization of the PEP amounts to use a code which has the maximum value of the minimum trace.

\section{BALANCED CODES}

\subsection{What is a "balanced code"?}

The concept of "balanced codes" has been proposed in [8-10]. It is based on the observation that the best STTCs proposed in the literature present the same property: the generated symbols of the MIMO constellation are equally probable.

The binary input data are supposed to be generated by a memory less binary source $S_{b}=\{0,1\}$ with equally probable symbols and $2^{n}$-PSK modulation are considered. From a given extended-state $\boldsymbol{X}=\left[x_{1} x_{2} \ldots x_{L}\right]^{T} \in \mathbb{Z}_{2}^{L}$ of the $L=n(v+1)$ length shift register realized by the input block of $n$ bits followed by the $v$ blocks of $n$ bits, the MIMO symbol $\boldsymbol{Y}=\left[y_{1} y_{2} \ldots y_{n_{T}}\right]^{T} \in \mathbb{Z}_{2^{n}}^{n_{T}}$ generated by the space time trellis encoder shown in Fig. 1 is

$$
\boldsymbol{Y}=\boldsymbol{G} \boldsymbol{X}
$$

where $\boldsymbol{G}$ is the generator matrix (1). This is a deterministic relation. Therefore, the STTC is defined by a map

$$
\Phi: \mathbb{Z}_{2}^{L} \rightarrow \mathbb{Z}_{2^{n}}^{n_{T}}
$$

which associates to the extended-state $\boldsymbol{X}$ a unique codeword $\boldsymbol{Y}$. Note that $\Phi\left(\mathbb{Z}_{2}^{L}\right) \subseteq \mathbb{Z}_{2^{n}}^{n_{T}}$ represents the set of generated codewords $\boldsymbol{Y}$.

By definition, a STTC is balanced if and only if each generated codeword $Y \in \Phi\left(\mathbb{Z}_{2}^{L}\right)$ has the same number of occurrences $n_{0} \in \mathbb{N}^{*}$.

The number of different codewords generated by relation (5) is given by $\operatorname{card}\left(\Phi\left(\mathbb{Z}_{2}^{L}\right)\right)$ which is a power of 2 .

The code is fully balanced if

$$
\left\{\sum_{j=1}^{j=L}\left(x_{j} G_{j}\right) \bmod 2^{n} / x_{j} \in\{0,1\}\right\}=\mathbb{Z}_{2^{n}}^{n_{T}}
$$

where $G_{j}$ is the $j^{\text {th }}$ column of the generator matrix $\boldsymbol{G}$. In this case: $\Phi\left(\mathbb{Z}_{2}^{L}\right)=\mathbb{Z}_{2^{n}}^{n_{T}}$.

Due to the random memory less source $S_{b}=\{0,1\}$, with $\mathrm{p}(0)=\mathrm{p}(1)=1 / 2$, from a given extended-state $\boldsymbol{X}$, the encoder has only $2^{n}$ equally probable next states. The matrix $\boldsymbol{T}$ of the transition probabilities between these states corresponds to a Markov chain. Due to the symmetry of the matrix $\boldsymbol{T}$, the steady-state probabilities of the extended-states $\boldsymbol{X}$ are all equal. For a balanced code, by using (5), the generated codewords $\boldsymbol{Y}$ are also equally-probable. In other words, the generated symbols of the MIMO constellation are equally probable.

\subsection{Properties of balanced STTC}

Theorem 1: If a STTC is fully balanced then $L \geq L_{\min }=n . n_{T}$. One can observe that $L_{\text {min }}=\operatorname{dim}\left(\mathbb{Z}_{2^{n}}^{n_{T}}\right)$. 
Theorem 2: Let us consider a fully balanced MIMO code with a $n_{T} \times L$ generator matrix $\boldsymbol{G}$. Then, for any additional column matrix $G_{L+1} \in \mathbb{Z}_{2^{n}}^{n_{T}}$ the resulting generator matrix $\boldsymbol{G}^{\prime}=\left[\boldsymbol{G} G_{L+1}\right]$ corresponds to a new fully balanced code.

Theorem 3: If $\boldsymbol{G}$ is the matrix of a balanced code, each permutation of its columns or/and lines generates the matrix of a new balanced code.

Theorem 4: $C_{[0]}=2^{n-1} \mathbb{Z}_{2}^{n_{T}}$ is a subgroup of $\mathbb{Z}_{2^{n}}^{n_{T}}$ such as $v=-v, \forall v \in C_{[0]}$. In the case of 4-PSK modulation, each element $v \in C_{[0]}$ can be written as $v=2 p$, with $p \in \mathbb{Z}_{2}^{n_{T}}$. The coset $C_{[p]}=p+C_{[0]}$ is called "relative to $v$ ". So, it is possible to write: $\mathbb{Z}_{4}^{n_{T}}=C_{[0]} \cup E_{1}$ where $E_{1}$ is the set of the cosets $C_{[p]} \neq C_{[0]}$. In the case of 8-PSK modulation, each element $v \in C_{[0]}=4 \mathbb{Z}_{2}^{n_{T}}$ can be written as $v=2 p$, with $p \in 2 \mathbb{Z}_{2}^{n_{T}}$. For 8-PSK, we note by $E_{1}$ the set of cosets which can be written as $C_{[p]}=p+C_{[0]}$ with $p \in 2 \mathbb{Z}_{2}^{n_{T}} \backslash\left\{\left[\begin{array}{ll}0 & 0\end{array}\right]^{T}\right\}$. So, each coset $C_{[p]}=p+C_{[0]} \in E_{1}$ is called "relative to $v$ ", with $v=2 p \in C_{[0]}$. Each element $q \in E_{1}$ can be written as $q=2 r$, with $r \in \mathbb{Z}_{4}^{n_{T}} \backslash 2 \mathbb{Z}_{2}^{n_{T}}$ and a new set $E_{2}$ of cosets $C_{[r]}=r+C_{[0]}$ can be created. Thus, each coset of $E_{2}$ is also "relative to an element of $E_{l}$ ". Therefore, it is possible to write: $\mathbb{Z}_{8}^{n_{T}}=C_{[0]} \cup E_{1} \cup E_{2}$.

\section{A NEW METHOD TO DESIGN $2^{n}$-PSK STTC WITH SEVERAL TRANSMIT ANTENNAS}

In [8-9], a first method to design fully balanced codes has been presented for several transmit antennas and 4-PSK STTC. In [10], a simpler method to design balanced 4-PSK STTC was presented. The general method described in this paper which is based on the method of [10] allows an easier design of balanced codes for any number of transmit antennas and for any $2^{n}$-PSK modulation. Due to Property 2 , it is enough to design fully balanced codes with $L=L_{\text {min }}$. This general method respects two rules:

- Rule 1: The first no null column $G_{1}$ must belong to $C_{[0]}$.

Due to the theorem $4, G_{1}=-G_{1}$, so $H_{1}=\left\{0, G_{1}\right\}$ is a subgroup of $\mathbb{Z}_{2^{n}}^{n_{T}}$.

- Rule 2: Each new chosen column of $\boldsymbol{G}$ must create a new subgroup $H_{i+1}$ of $\mathbb{Z}_{2^{n}}^{n_{T}}$ with $\operatorname{card}\left(H_{i+1}\right)=2 \operatorname{card}\left(H_{i}\right)$.

Thus, in order to obtain a fully balanced STTC, this general algorithm is as follows:

if $i<L_{\min }$ columns of $\boldsymbol{G}$ have been already chosen in $\mathbb{Z}_{2^{n}}^{n_{T}}$ and $\left\{\sum_{j=1}^{i} x_{j} G_{j} \bmod 2^{n} / x_{j} \in\{0,1\}\right\}=H_{i}$ is a subgroup of $\mathbb{Z}_{2^{n}}^{n_{T}}$, then the column $G_{i+1}$ of $\boldsymbol{G}$ must belong to $\mathbb{Z}_{2^{n}}^{n_{T}} \backslash H_{i}$ and must be selected in the cosets relative to the vectors which belong to $H_{i}$ or in the coset $C_{[0]}$. This method ensures that $H_{i+1}=H_{i} \cup\left(H_{i}+G_{i+1}\right)$ is a subgroup of $\mathbb{Z}_{2^{n}}^{n_{T}}$.

The algorithm ends when $i+1=L_{\min }$. The obtained matrix $\boldsymbol{G}$ corresponds to a fully balanced code with $n_{0}=1$. If necessary, more columns can be added to $\boldsymbol{G}$ to create a fully balanced code with $n_{0}>1$.

In order to obtain balanced codes (not necessary fully balanced) the algorithm can be ended when $H_{i 0}$ is created with $i_{0} \leq L_{\text {min }}-2$ columns. One can add a new column $G_{i_{0}+1} \in \mathbb{Z}_{2^{n}}^{n_{T}} \backslash H_{i_{0}}$. Once again, the obtained code is balanced but not fully balanced. If necessary, several elements of $H_{i_{0}}$ can also be added as columns of the matrix $\boldsymbol{G}$. The resulting code is also balanced but not fully balanced.

Remark: The main idea of this new method is to generate at each step several new elements of $\mathbb{Z}_{2^{n}}^{n_{T}}$ and their opposites. Therefore, there are not discarded elements of $\mathbb{Z}_{2^{n}}^{n_{T}}$ (elements that must not be further selected). Thus, this new method to design the B-STTC is simpler than the method described in [89].

\subsection{Design of fully balanced 4-PSK STTC with 2 transmit antennas}

In this case, each generated element belongs to $\mathbb{Z}_{4}^{2}$. It is possible to make the partition in cosets: $C_{[p]}=p+C_{[0]}$ with $C_{[0]}=2 \mathbb{Z}_{2}^{2}$ and $p \in \mathbb{Z}_{2}^{2}$. Thanks to this partition, to generate a balanced code with $L_{m i n}=4$ columns, we can proceed as follows:

- In agreement with rule 1 , the first column $G_{1}$ of $\boldsymbol{G}$ must be selected in $C_{[0]}$ (but $G_{1} \neq\left[\begin{array}{l}0 \\ 0\end{array}\right]$ ), because $C_{[0]}$ contains the vectors with the property : $G_{i}=-G_{i}$. Therefore, a subgroup of $\mathbb{Z}_{4}^{2}$ is generated: $H_{1}=\left\{0, G_{1}\right\}$.

- The second element $G_{2}$ has to create a new subgroup $H_{2}=\left\{0, G_{1}, G_{2}, G_{1}+G_{2}\right\}$, where $-G_{1},-G_{2}$ and $-\left(G_{1}+G_{2}\right)$ are included in $H_{2}$. There are two solutions to choose $G_{2}$ :

- If $G_{2} \in C_{[0]} \backslash H_{1}$, then $-G_{2}=G_{2},-\left(G_{1}+G_{2}\right)=G_{1}+G_{2}$ and $H_{2}=\left\{0, G_{1}, G_{2}, G_{1}+G_{2}\right\}=C_{[0]}$ is a subgroup of $\mathbb{Z}_{4}^{2}$. This type of code is called [8, 10] Type II, i.e. $\boldsymbol{G}$ has 2 columns in $C_{[0]}$ and thus $C_{[0]}$ is totally generated by $G_{1}$ and $G_{2}$. It remains to select two different cosets and in each coset, one vector must be selected. In fact, because $G_{3} \in \mathbb{Z}_{4}^{2} \backslash C_{[0]}, H_{3}=C_{[0]} \cup\left(C_{[0]}+G_{3}\right)$ is a new 
subgroup of $\mathbb{Z}_{4}^{2}$. Finally, if $G_{4} \in \mathbb{Z}_{4}^{2} \backslash H_{3}$, then $H_{4}=H_{3} \cup\left(H_{3}+G_{4}\right)=\mathbb{Z}_{4}^{2}$.

- If $G_{2} \in C_{\left[p_{1}\right]}$ with $2 p_{1}=G_{1}$, then $G_{1}+G_{2}=-G_{2}$ and $H_{2}=\left\{0, G_{1}, G_{2}, G_{1}+G_{2}\right\}$ is a subgroup of $\mathbb{Z}_{4}^{2}$. If no more vector is selected in $C_{[0]}$, this type of code is called $[8,9]$ Type $I$. To create a new group $H_{3}=H_{2} \cup\left(H_{2}+G_{3}\right)$ without using a new vector of $C_{[0]}$ (else the generator matrix $\boldsymbol{G}$ would correspond to a balanced code of type II), the third column of $\boldsymbol{G}$ must be selected in $C_{\left[p_{1}\right]}$ among the two elements that are not generated by the combinations of $G_{l}$ and $G_{2}$. So, thanks to the combinations between $G_{1}, G_{2}$ and $G_{3}$, the group $H_{3}$ is equal to $C_{[0]} \cup C_{\left[p_{1}\right]}$. The last vector $G_{4}$ is selected among the two non generated cosets. Then, $\left\{G_{l}, \ldots G_{4}\right\}$ is a base of $\mathbb{Z}_{4}^{2}$ and thus a fully balanced space-time trellis code for 4-PSK modulation and 2 transmit antennas is obtained.

\subsection{Design of fully balanced 8-PSK STTC with $n_{T}$ transmit antennas}

For 8-PSK STTC with 2 transmit antennas, each generated element belongs to $\mathbb{Z}_{8}^{n_{T}}$. In agreement with theorem 4 , it is possible to make the partition of the set of the cosets: $\mathbb{Z}_{8}^{n_{T}}=C_{[0]} \cup E_{1} \cup E_{2}$ where each coset of $E_{2}$ is relative to one element of $E_{1}$ and each coset of $E_{1}$ is relative to one element of $C_{[0]}$. Thanks to this partition, in order to generate a balanced code, we can proceed as follows:

- In agreement with rule 1 , the first column $G_{l}$ of $\boldsymbol{G}$ must be selected in $C_{[0]}$ (but $G_{1} \neq\left[\begin{array}{lll}0 & \cdots & 0\end{array}\right]^{T}$ ), because $C_{[0]}$ contains the vectors with the property : $G_{i}=-G_{i}$. Therefore, a subgroup of $\mathbb{Z}_{4}^{2}$ is generated: $H_{1}=\left\{0, G_{1}\right\}$.

- The $i^{\text {th }}$ column $G_{i}(\mathrm{i}>1)$ can be selected either :

- in $C_{[0]} \backslash H_{i-1}$.

- or in the coset $C_{[p]} \in E_{1} \backslash H_{i-1}$ if and only if $2 p \in H_{i-1}$.

- or in the coset $C_{[q]} \in E_{2} \backslash H_{i-1}$ if and only if $2 q \in H_{i-1}$.

Thus, each new selected column creates a new subgroup with twice more elements than the previous subgroup.

\section{PERformanCE OF NEW CODES}

In Table 1, the minimum trace of some new balanced codes are compared to the minimum trace of Chen's codes with 3 and 4 transmit antennas. These codes have 32 and 64 states. The new codes have higher values of the minimum trace than the traces of Chen's equivalent codes. The codes noted by
' $\mathrm{B}$ ' are balanced, those by ' $\mathrm{FB}$ ' are fully balanced and those noted by 'NB' are not balanced. Each new code is balanced and its trace is greater than the trace of Chen's corresponding code.

TABLE 1: 4-PSK Balanced STTCs

with 3 and 4 transmit antennas

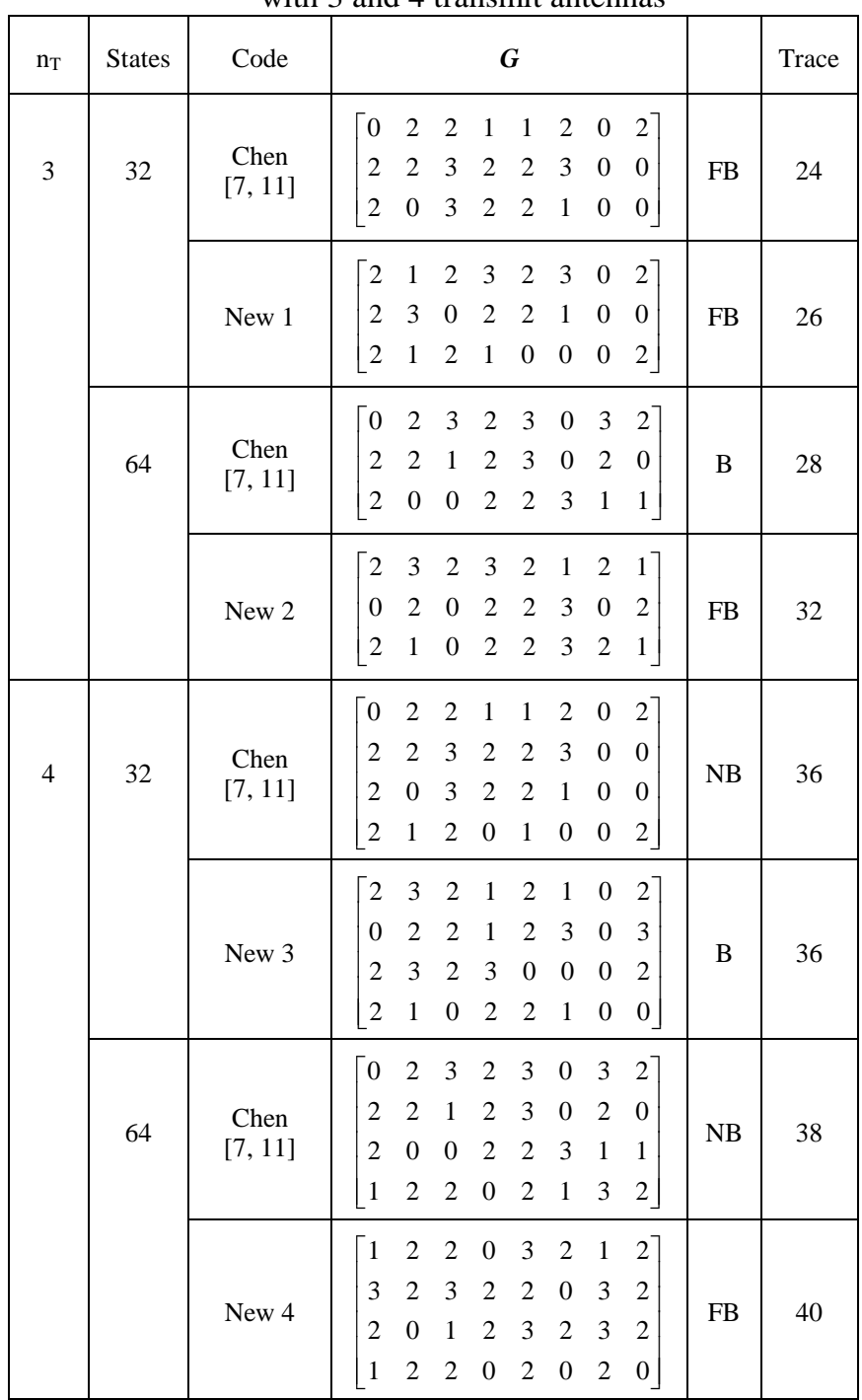

In Table 2, 8-PSK Chen's codes and new corresponding codes are shown for 3 transmit antennas/16 states and 4 transmit antennas/8 states. The two new codes have higher values of the minimum trace than the traces of Chen's equivalent codes.

The performance of each code is evaluated by simulation in a slow Rayleigh fading channel. The channel fading coefficients are independent samples of a complex Gaussian process with zero mean and variance 0.5 per dimension. These channel coefficients are assumed to be known at the decoder. Each frame consists of 130 4-PSK symbols or 198 8-PSK symbols. For the simulation, there are 2 receive antennas. The decoding is performed by the Viterbi's algorithm. Figure 2 shows the performance of the 4-PSK 32 states and 64 states codes for 3 transmit antennas given in Table 1 . In the same way, the performance of 4-PSK STTC with 32 states and 64 
states for 4 transmit antennas is shown in figure 3 . Figure 4 shows the performance of the new 8-PSK and the corresponding Chen's codes of Table 2.

TABLE 2: 8-PSK Balanced STTCs with 3 and 4 transmit antennas

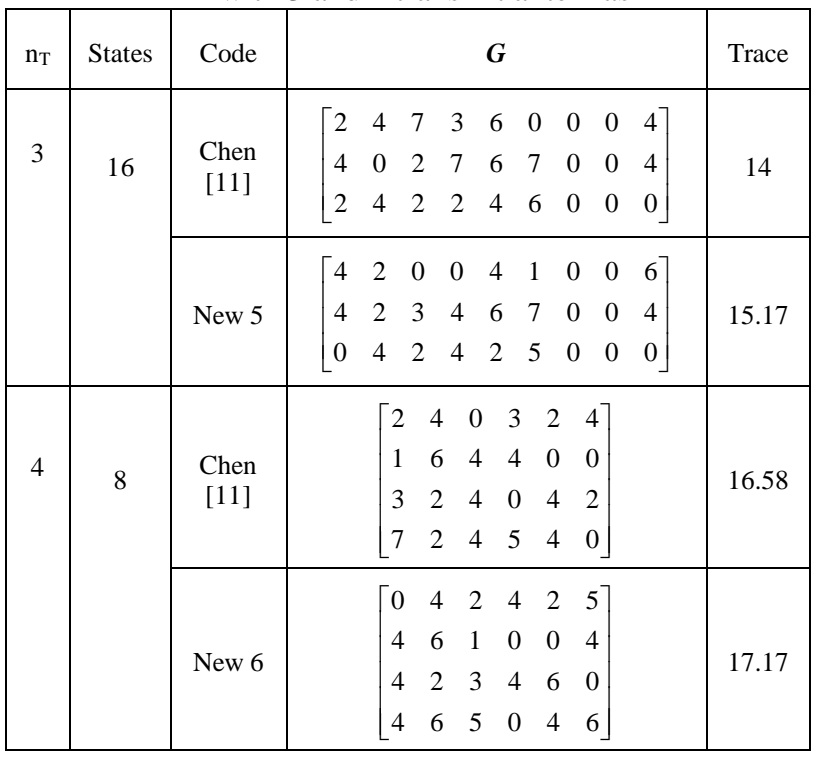

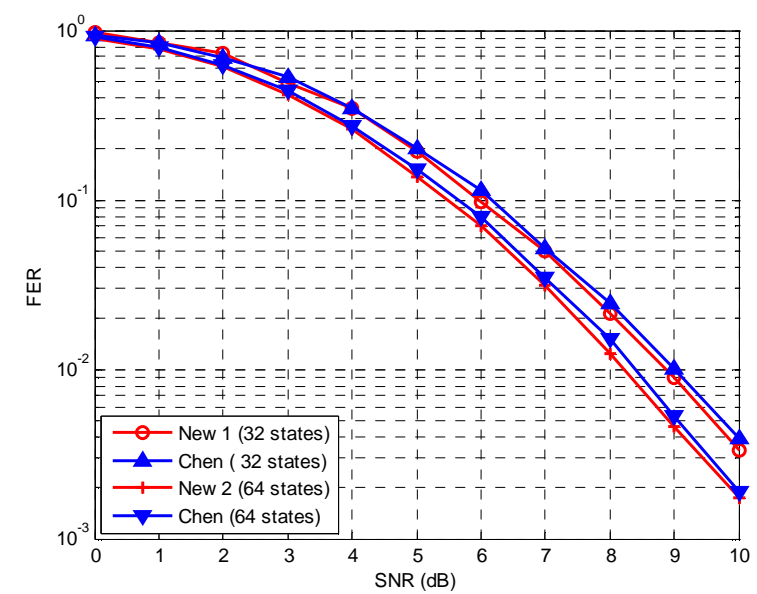

Fig. 2. Performance of 4-PSK STTC with 3 Tx / 2 Rx antennas

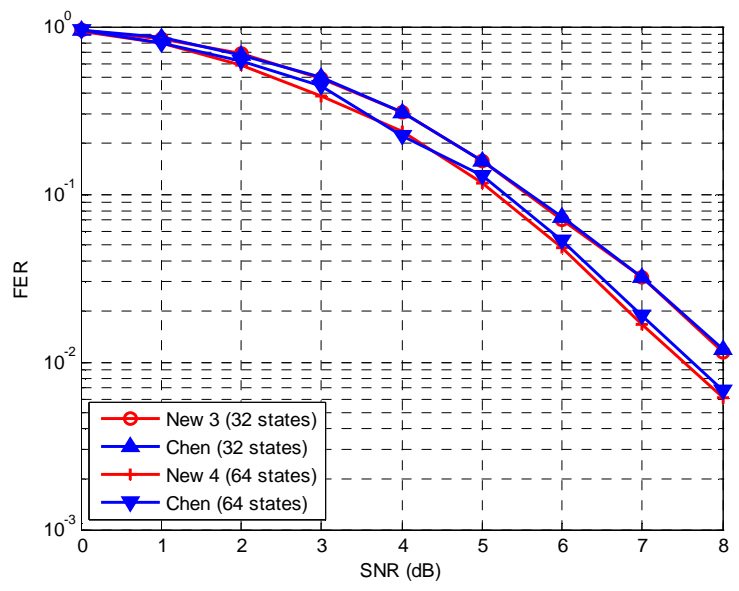

Fig. 3. Performance of 4-PSK STTC with $4 \mathrm{Tx} / 2 \mathrm{Rx}$ antennas

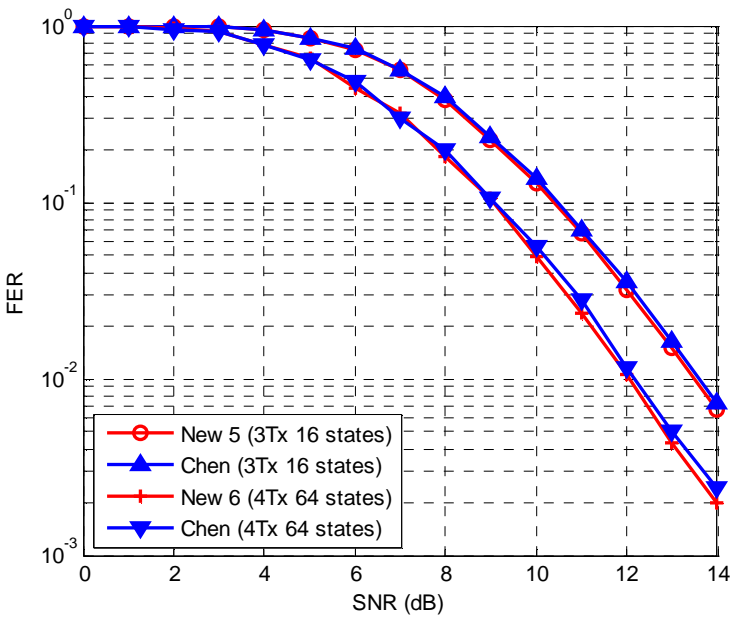

Fig. 2. Performance of 8-PSK STTC with 3 Tx \& 4 Tx / 2 Rx antennas

\section{CONCLUSION}

This paper presents a new and simpler method to design balanced space-time trellis codes. It has been shown that the best codes belong to this class. The balanced codes generate the points of the MIMO constellation with the same probability. This new and general method allows to design the balanced STTCs for $2^{n}$-PSK modulations and $n_{T}$ transmit antennas. The search for the best codes can be reduced to the class of balanced codes. Furthermore, several new balanced 4PSK and 8-PSK codes for 3 and 4 transmit antennas which outperform the best previously published codes have also been proposed.

\section{REFERENCES}

[1] V. Tarokh, N. Seshadri, and A.R. Calderbank, "Space-time codes for high data rate wireless communication: Performance criterion and code construction," IEEE Trans. Inform. Theory, vol. 44, no. 2, pp. 744-765, March 1998.

[2] Z. Chen, J. Yuan, and B. Vucetic, "Improved space-time trellis coded modulation scheme on slow fading channels," Electron. Lett., vol. 37, no. 7, pp. 440-441, March 2001 .

[3] W. Firmanto, B. Vucetic, and J. Yuan, "Space-time TCM with improved performance on fast fading channels," IEEE Commun. Lett., vol. 5, no. 4, pp. 154-156, April 2001.

[4] S. Baro, G. Bauch, and A. Hansmann, "Improved codes for space-time trellis-coded modulation," IEEE Commun. Lett., vol. 4, no. 1, pp. 20-22, January 2000.

[5] Yi Hong and A. Guillen i Fabregas, "New Space-Time Trellis Codes for Slow Fading Channels," in Proc. IEEE VTC 2006-Spring, vol. 3, pp. 14921496, May 2006.

[6] Y.S. Jung and J.H. Lee, "Improved space-time trellis codes using expanded signal set in slow Rayleigh fading channels," in Proc. IEEE ISIT'2002, p. 248, June 2002.

[7] Z. Chen, B. Vucetic, J. Yuan, and Ka Leong Lo, "Space-time trellis codes for 4-PSK with three and four transmit antennas in quasi-static flat fading channels," IEEE Commun. Lett., vol. 6, no. 2, pp. 67-69, Feb. 2002.

[8] T. M. H. Ngo, G. Zaharia, S. Bougeard and J.F. Helard, "A new class of balanced 4-PSK STTC for two and three transmit antennas," in Proc. IEEE 8th Workshop on Signal Processing Advances in Wireless Communications, SPAWC 2007, pp. 1-5, June 2007.

[9] T. M. H. Ngo, P. Viland, G. Zaharia, and J.F. Helard, "Balanced QPSK space-time trellis codes", Electron. Lett., vol. 44, no. 16, July 2008.

[10] P. Viland, G. Zaharia, J.F. Helard, "An improved method to design QPSK Balanced Space-Time Trellis Codes", in Proc. IEEE 14th European Wireless Conference 2008 (EW2008), pp. 1-6, 22 - 25 June 2008.

[11] B. Vucetic, J. Yuan “Space-Time Coding," Wiley, 2003. 